Methods In a prospective study among 2269 HIV-1 infected ARTnaïve women from 7 countries in East and southern Africa, we examined the effect of pregnancy on HIV-1 disease progression. We used random effects models to compare CD4 and plasma viral load changes between pregnant, postpartum and non-pregnant periods (prenatal periods from women who became pregnant and all periods from women who did not become pregnant). Among women who became pregnant, we compared CD4 counts during prenatal, pregnant, and postpartum periods.

Results Women contributed 3471 person-years and 475 women became pregnant $(7.2 \%$ of time was pregnant and $6.8 \%$ was postpartum). After accounting for baseline levels, CD4 counts were 67.7 cells/ $\mathrm{mm}^{3}$ lower (95\% CI 55.5-79.9) during pregnant compared to nonpregnant periods and 81.2 cells $/ \mathrm{mm}^{3}$ lower (95\% CI 65.3-97.2) during pregnant compared to postpartum periods. After adjustment for baseline viral load, there were small increases in plasma viral load: a 0.05 $\log _{10}$ increase in pregnant vs. non-pregnant periods (95\% CI 0.01-0.10) and a $0.08 \log _{10}$ increase in pregnant vs. postpartum periods $(95 \% \mathrm{CI}$ $0.01-0.14)$. Postpartum CD4 and plasma viral loads were not different from non-pregnant periods $(p=0.1$ and $p=0.5)$. Among women who experienced pregnancy, CD4 counts were 59.6 cells $/ \mathrm{mm}^{3}$ lower $(95 \%$ CI 35.2-84.0) during pregnant versus prenatal periods and 71.6 cells/ $\mathrm{mm}^{3}$ lower (95\% CI 48.0-95.1) during pregnant versus postpartum periods. Prenatal and postpartum CD4 counts were similar $(p=0.4)$.

Conclusion CD4 count and plasma viral load changes among HIV-1 infected women during pregnancy are not permanent and are likely to return to prenatal levels. Pregnancy was not associated with subsequent disease progression.

\section{P3.201 RECENT SYPHILIS PREDICTS HEPATITIS C VIRUS (HCV) SEROCONVERSION AMONG HIV-POSITIVE MEN WHO HAVE SEX WITH MEN (MSM)}

doi:10.1136/sextrans-2013-051184.0658

\begin{abstract}
${ }^{1,2}$ A N Burchell, 'M Manno, ${ }^{1,2} \mathrm{~S}$ Gardner, ${ }^{3} \mathrm{~T}$ Mazzulli, ${ }^{2} \mathrm{R}$ S Remis, ${ }^{4,2} \mathrm{~A}$ Bayoumi, ${ }^{5} \mathrm{~F}$ McGee, ${ }^{2} \mathrm{P}$ Millson, ${ }^{6,2} \mathrm{~J}$ Raboud, ${ }^{1,2} \mathrm{~S}$ B Rourke. ${ }^{1}$ Ontario HIV Treatment Network, Toronto, ON, Canada; ${ }^{2}$ University of Toronto, Toronto, ON, Canada; ${ }^{3}$ Public Health Ontario, Toronto, ON, Canada; ${ }^{4}$ St. Michael's Hospital, Toronto, ON, Canada; ${ }^{5}$ Ontario Ministry of Health and Long Term Care, Toronto, ON, Canada; ${ }^{6}$ University Health Network, Toronto, ON, Canada
\end{abstract}

Background There is evidence of sexual HCV transmission among HIV-positive MSM from the UK and Europe. We estimated HCV seroincidence and its risk factors in a North American population of HIV-positive MSM with no known history of injection drug use.

Methods We analysed data from the OHTN Cohort Study, an ongoing cohort of persons in HIV care in Ontario, Canada. Data were obtained from medical charts, interviews, and record linkage with the provincial public health laboratories. We restricted the analysis to 1,534 MSM who: (1) did not report injection drug use; (2) were under follow-up in 2000-2010; and (3) had 2+ HCV antibody tests, of which the first was negative. Person-time commenced at the later of the HCV-negative result or HIV diagnosis and ended at the first $\mathrm{HCV}+$ or last date of follow-up (median 6.1 person-years (PY) of follow-up; sum 9,987PY).

Results We observed $51 \mathrm{HCV}$ seroconversions, for an overall incidence of 0.51 per 100PY (CI: 0.39-0.67). Annual incidence varied from 0.16 to 0.89 per 100 PY, with no statistical evidence of a temporal trend. Seroconversion was statistically-significantly associated with acute syphilis infection in the previous 6 months (adjusted hazard ratio $=4.9, \mathrm{CI} 1.2-21$ ) and there was a marginally statistically-significant association for men who had not yet initiated antiretroviral treatment (adjusted hazard ratio $=1.9$, CI 0.914.0). There were no statistically significant effects of age, ethnicity, region, CD4+ cell count or viral load.
Conclusion Sexual behaviour was unmeasured and we cannot exclude the possibility of HCV acquisition via unreported injection drug use. Nevertheless, the strong association with recent syphilis suggests that at least some cases were due to sexual transmission. Future research is needed to establish whether syphilis is a marker for high-risk behaviour or may potentiate sexual HCV transmission among persons with HIV.

\section{P3.202 ESTIMATION OF HIV INCIDENCE IN BRAZIL, 2004-2011}

doi:10.1136/sextrans-2013-051184.0659

C L Szwarcwald, P R B Souza-Jr. Fiocruz, Rio de Janeiro, Brazil

Background The HIV incidence is the most valuable indicator of epidemiological surveillance as it establishes the degree to which HIV transmission is occurring and which groups are most at risk for HIV infection. However, the HIV incidence is a difficult indicator to estimate. This paper addresses a method to estimate the HIV incidence in Brazil in recent years.

Methods The information source is SISCEL, which is the national laboratory-based surveillance system created to monitor CD4+/ CD8+ T lymphocytes and HIV viral load. The proposed method is based on the first CD4 count after HIV diagnosis among all treatment-naïve cases registered in SISCEL in the time period 2004-2011. A regression model that relates progress of CD4 to time of seroconversion was used to estimate time of HIV infection at the date of first CD4 count. The analysis was performed by sex and age group. Results For all years, the proportion of HIV cases registered in SISCEL in the same year of infection was $31 \%$, and approximately $6 \%$ for each year of the 5 subsequent years after infection, so that almost $40 \%$ cases are registered in SISCEL only 7 years or more after infection. The HIV incidence was stable in the period 2005-2011. After adjusting for HIV cases tested in private laboratory and undiagnosed cases not registered in SISCEL, the mean estimate was 41600 cases, corresponding to an HIV incidence rate of 0.27 per 1000 population. Analysis by sex and age showed a rising trend among males, particularly for young men, and a decreasing trend among females.

Conclusion In terms of late HIV diagnosis, the results indicate that coverage of HIV testing in the general population should be largely expanded. As to most at risk populations, the findings suggest that interventions should be focused on young adult men to reverse the increasing trend.

\section{P3.203 MORTALITY RATES IN HIV-1/2 DUAL- AND MONO-INFECTED INDIVIDUALS: A SYSTEMATIC REVIEW AND META- ANALYSIS}

doi:10.1136/sextrans-2013-051184.0660

'P Prince, ${ }^{1} \mathrm{~A}$ Matser, ${ }^{2} \mathrm{C}$ van Tienen, ${ }^{1} \mathbf{M}$ Schim van der Loeff. ${ }^{\text {P }}$ ublic Health Service of Amsterdam, Amsterdam, The Netherlands; ${ }^{2}$ Medical Microbiology \& Infectious Diseases, Erasmus Medical Center, Rotterdam, The Netherlands

Background Although HIV-1 and HIV-2 share the same transmission routes, HIV-2 is less transmissible, it has a longer median time from infection to AIDS, and the associated mortality risk is lower. It has been suggested that HIV-2 infection inhibits HIV-1 disease progression in dually infected (HIV-D) individuals, but whether the mortality rate of HIV-D infected individuals differs from that of HIV-1 mono-infected individuals is still not clear. We conducted a systematic review and meta-analysis on HIV mortality.

Methods Medline and Embase databases were searched. The inclusion criteria for studies were an antiretroviral therapy-naïve population during follow-up, reporting mortality data and 